\title{
A Compact Dual-Band Printed Antenna Design for LTE Operation in Handheld Device Applications
}

\author{
Ding-Bing Lin, ${ }^{1}$ Jui-Hung Chou, ${ }^{2}$ Son-On Fu, ${ }^{3}$ and Hsueh-Jyh $\mathrm{Li}^{4}$ \\ ${ }^{1}$ Department of Electronic Engineering, National Taipei University of Technology, Taipei 10608, Taiwan \\ ${ }^{2}$ Graduate Institute of Communication Engineering, National Taiwan University, Taipei 10617, Taiwan \\ ${ }^{3}$ Graduate Institute of Computer and Communication Engineering, National Taipei University of Technology, Taipei 10608, Taiwan \\ ${ }^{4}$ Department of Electrical Engineering and the Graduate Institute of Communication Engineering, National Taiwan University, \\ Taipei 10617, Taiwan
}

Correspondence should be addressed to Jui-Hung Chou; d98942001@ntu.edu.tw

Received 7 March 2014; Revised 23 May 2014; Accepted 24 May 2014; Published 18 June 2014

Academic Editor: Yingsong Li

Copyright (C) 2014 Ding-Bing Lin et al. This is an open access article distributed under the Creative Commons Attribution License, which permits unrestricted use, distribution, and reproduction in any medium, provided the original work is properly cited.

A novel internal printed antenna suitable for triple long-term evolution (LTE) bands for handheld devices is presented. The operating bandwidths of the design are LTE700 $(698 \sim 800 \mathrm{MHz})$, LTE2300 $(2300 \sim 2400 \mathrm{MHz})$, and LTE2500 $(2500 \sim 2690 \mathrm{MHz})$. Through the use of a C-shape broadside coupled feed structure, full operation in the lower band is achieved. The antenna itself uses two unequal path lengths to produce a low frequency band with two resonant modes. The required bandwidth is then adjusted using a couple feed, and finally placed over a ground plane via another C-type coupling element in order to enhance the two lowfrequency matches. In the definition of the $-6 \mathrm{~dB}$ reflection coefficient, the bandwidth of two basic modes in the low frequency band is $0.689 \sim 0.8 \mathrm{GHz}$. We adopt the definition of the $-10 \mathrm{~dB}$ reflection coefficient for the high frequency mode, and its working frequency bands are shown to be $2.3 \sim 2.72 \mathrm{GHz}$. The antenna size is only $40 \times 12 \times 0.8 \mathrm{~mm}^{3}$ with a ground plane of $98 \times 40 \mathrm{~mm}$.

\section{Introduction}

In recent years, mobile communication requires a handheld mobile device to function on multiple communication systems which has made the multiband mobile antenna increasingly important [1-3]. In view of the growth of consumer demands for speed and bandwidth, the fourth generation of long-term evolution (LTE) mobile communication technology has been developed. However, the requirement for lower and wider frequency bands has increased the difficulty of antenna design. The bandwidth achievable from a conventional planar antenna is often insufficient. Although the use of $3 \mathrm{D}$ concepts is able to increase bandwidth, this approach also increases the thickness of the resulting device. In [4], a planar structure was used to design an antenna functioning in the LTE700/LTE2300/LTE2500 bands. Although the design was capable of covering whole frequency bands, it required a relatively large surface area for its implementation. In [5], a relatively smaller planar structure was used to design a multiband antenna, which also covered the frequency bands of LTE700/LTE2300/LTE2500. However, in this case the low frequency section failed to cover the entire band; it narrowed the relative bandwidth of the planar structure covering the same area as the 3D structure. This is because a 3D structure can often use its side to widen the wire and decrease the $Q$ value in order to increase its operational bandwidth. In [6], the use of a magnetic material substrate overcame the observed shortcomings. The magnetic permeability from the magnetic material significantly reduced the electrical length of the low frequency band. However, it simultaneously increased hysteresis loss and decreased the gain. In addition, high-quality factors resulted in an insufficient bandwidth. A multiband loop antenna was proposed in [7]. The antenna structure is relatively large, yet the design easily meets the required bandwidth. The antenna was designed by observing the structure of the antenna current density in order to widen where the density is weak to achieve a broader band.

This paper presents a miniaturized printed antenna design for a mobile communication device, which operates at frequency bands meeting the required operating bandwidths 


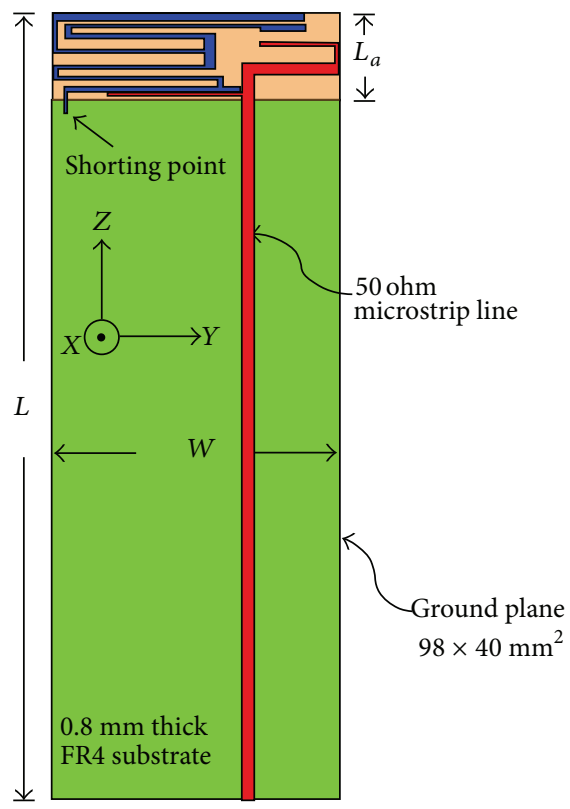

(a)

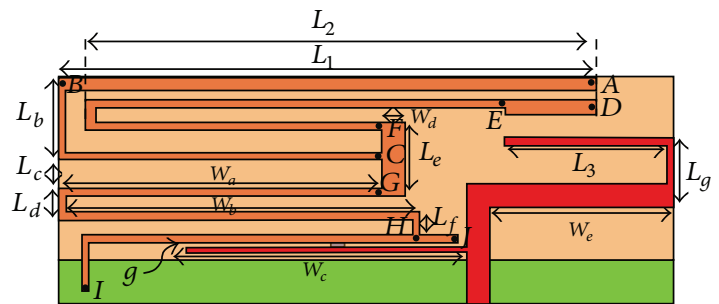

(b)

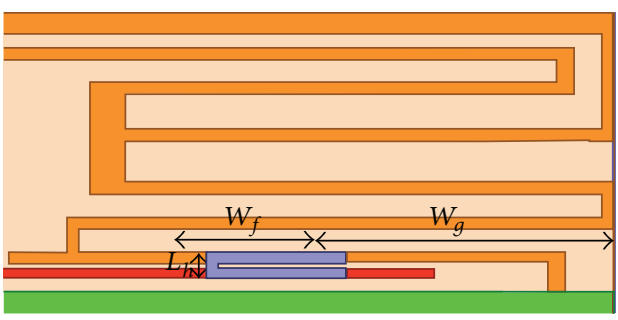

(c)

FIGURE 1: The geometry of the proposed compact printed antenna (a) with system ground, (b) top view, and (c) bottom view.

of LTE700, LTE2300, and LTE2500. The antenna design uses a base band and second harmonic in combination with different resonance paths to achieve the desired operating bandwidths. Through the use of a coupling feed and imbedding a C-shape coupler underneath, the proposed antenna achieves excellent impedance matching within the operating bands.

\section{Proposed Antenna Design}

The overall structure of the proposed LTE concealed multiband mobile communication antenna is shown in Figure 1. The antenna is printed on a FR4 substrate with a thickness of $0.8 \mathrm{~mm}$, permittivity of 4.4 , and tangent loss of 0.025 . The overall size of the antenna and handheld device is $110 \times 40$ $\times 0.8 \mathrm{~mm}^{3}$, where the antenna size is $12 \times 40 \times 0.8 \mathrm{~mm}^{3}$ and has a ground plane of $98 \times 40 \mathrm{~mm}^{2}$. The antenna is fed by a microstrip line with a characteristic impedance of $50 \mathrm{ohms}$ and width of $1.5 \mathrm{~mm}$. The geometric structure and the detailed physical size of the LTE multiband mobile communication antenna design of this paper are shown in Figure 1(b).
In Figure 1(b) the top view of the antenna radiating body is shown. The antenna mainly contains two radiation paths, which are labeled (A-I) and (D-I); the two paths of resonant frequencies generate the required operating bandwidth for the LTE700 frequency band. The physical length is approximately two resonance frequencies of $1 / 4 \lambda_{g}$ of the required resonant length. In Figure 1(c) the bottom view of the antenna is shown. We used a C-type coupler in the back of the antenna. The detailed dimensions of the parameters are listed in Table 1. The parameters and dimensions of the proposed antenna are achieved by using the full-wave electromagnetic simulator, which is Ansoft high frequency structure simulator (HFSS) [8]. The antenna adopts a coupling feed structure, which can increase the capacitance effects from the antenna feeding to improve the inductive effects produced by the thin metal wire. It can significantly improve input impedance of the antenna and make it easy to match the characteristic impedance of the system. However, since the design of this antenna is limited by the size, it is not suitable to use the traditional interdigital coupling feed structure to generate enough capacitance. Therefore, by imbedding a C-type coupler behind the original coupling feed we can 
TABLE 1: The detailed dimension of the parameters in Figure 1.

\begin{tabular}{|c|c|c|c|c|c|}
\hline Parameter & Length (mm) & Parameter & Length (mm) & Parameter & Length $(\mathrm{mm})$ \\
\hline$\overline{\overline{\mathrm{AC}}}$ & 61.5 & $L$ & 110 & $W$ & 40 \\
\hline$\overline{\mathrm{DE}}$ & 6 & $L_{a}$ & 12 & $W_{a}$ & 21 \\
\hline$\overline{\mathrm{EF}}$ & 47 & $L_{b}$ & 5.5 & $W_{b}$ & 23.5 \\
\hline$\overline{\mathrm{GH}}$ & 46.75 & $L_{c}$ & 1.8 & $W_{c}$ & 17 \\
\hline$\overline{\mathrm{HI}}$ & 24.7 & $L_{d}$ & 2 & $W_{d}$ & 1.52 \\
\hline$L_{1}$ & 36 & $L_{e}$ & 4.8 & $W_{e}$ & 12 \\
\hline$L_{2}$ & 34 & $L_{f}$ & 1.5 & $W_{f}$ & 6 \\
\hline$L_{3}$ & 10.5 & $L_{g}$ & 4.5 & $W_{g}$ & 11.5 \\
\hline$g$ & 0.2 & $L_{h}$ & 1.1 & & \\
\hline
\end{tabular}

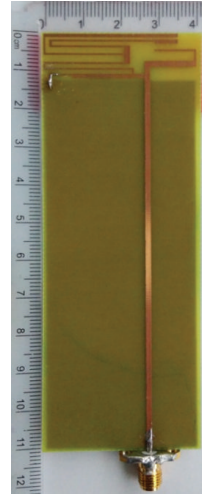

(a)

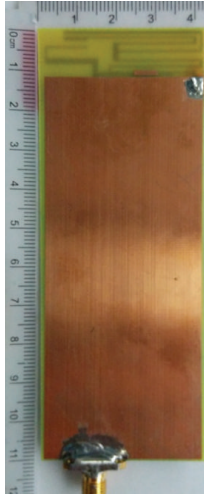

(b)
FIGURE 2: Experimental prototype of the proposed compact printed antenna (a) top view and (b) bottom view.

dramatically increase (and, in conjunction, improve) the capacitance effects of the required antenna matching. The structure of the coupling feed will be analyzed in detail in the next paragraph.

\section{Results and Discussions}

3.1. Resonance Mechanism of the Proposed Antenna. In order to measure the proposed antenna characteristics, we use an SMA connector with a $50 \mathrm{ohm}$ characteristic impedance in the actual experiments. Its center conductor is connected to the microstrip line, and the outer ground is connected directly to the system ground plane. In Figure 2 the experimental prototype is shown, while Figure 3 shows the simulated and measured reflection coefficients. The simulated and measured antenna reflection coefficients are in agreement, albeit with a minor variation at the edge of the frequency band caused by inaccuracies in the material when actually fabricating the plates. In the low-frequency part, defined by a $-6 \mathrm{~dB}$ reflection coefficient, the operating bandwidth can be up to $110 \mathrm{MHz}(690 \sim 800 \mathrm{MHz})$, which contains the LTE 700 full-band operation required bandwidth. In the high-frequency part, using the $-10 \mathrm{~dB}$ reflection coefficient definition, it can cover the entire required frequency of the LTE2300/2500 operating bands. The antenna measurement is seen to reach a high-frequency bandwidth up to $410 \mathrm{MHz}$ (2300 2710 MHz).

In order to accelerate the analysis of the antenna radiation characteristics and reduce the material waste caused by constant implementation, we used the HFSS [8] to analyze the relevant characteristics of the antenna. The antenna current distribution determined by the simulation is shown in Figure 4, which is obtained from the observation of each band in Figure 3. In the observed frequency ranges, the four resonant frequency points are $724,774,2320$, and $2550 \mathrm{MHz}$, which correspond to the simulated current distribution as shown in Figures 4(a) 4(d). We first observed the current distribution of the two low frequencies of the resonant frequency points. In order to meet the resonance boundary condition of short to open circuit, the main resonant path is approximately a physical length of $1 / 4 \lambda_{g}$ of the resonant frequency. To observe the current distribution of the first resonant frequency point in the high-frequency band, the main resonance frequency mechanism is the base frequency multiplication of the low resonant path. Therefore, by observing the current distribution we can witness the current null point generated from the resonance. To observe the current distribution of the second resonant frequency point in the high-frequency band, the main current distribution is concentrated on the right side of the conductor and the distribution is approximately $1 / 4 \lambda_{g}$ of the monopole antenna.

3.2. Parametric Analysis. As mentioned above, due to a limited design space, this antenna cannot use an interdigital coupling feed to dramatically increase the capacitance effect. Therefore, a C-shape coupler was added behind the parallelcoupled feed line to increase the required capacitive effect for impedance matching and improve the matching within the antenna operational band. In Figure 5 the simulated reflection coefficient graph of the antenna embedding with and without the C-type coupler is shown. The embedded C-type coupler under the coupling feed line improves the antenna's impedance characteristics. Specifically, in the low frequency range, without the coupler, the matching condition on the conjunction of the two resonant frequency points is not satisfied; however, with the addition of the coupler, the impedance characteristics of the low frequency band are significantly improved and can easily meet the $-6 \mathrm{~dB}$ reflection coefficient definition. 


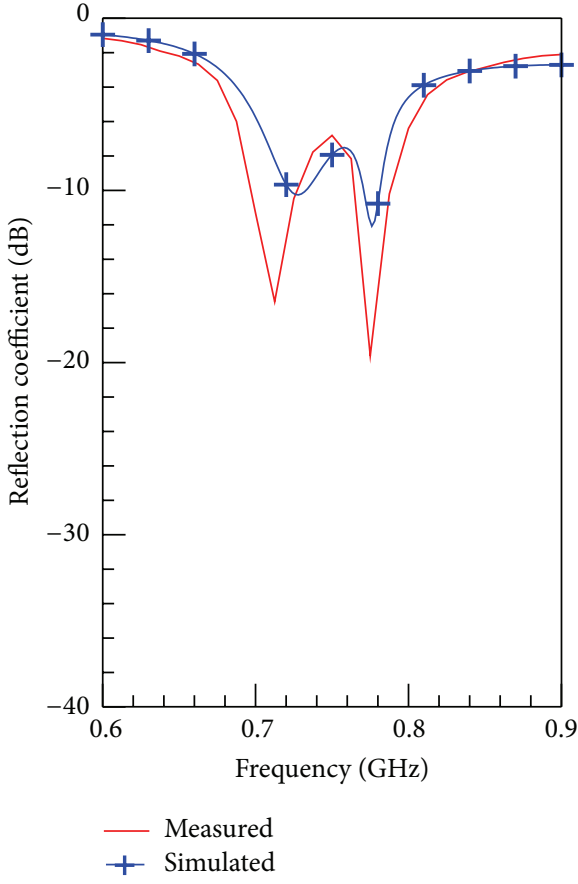

(a)

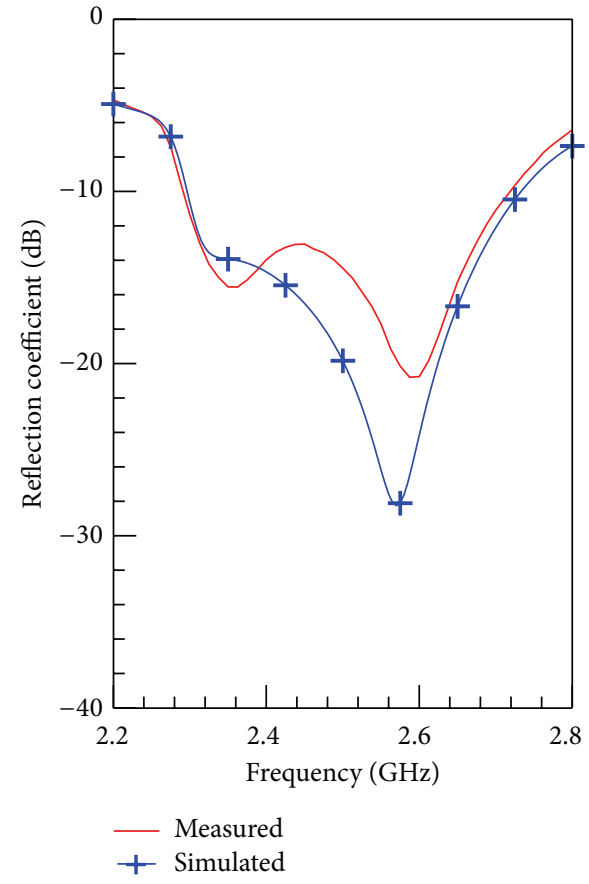

(b)

FIGURE 3: Measured and simulated reflection coefficient of the proposed miniaturized antenna versus frequency.

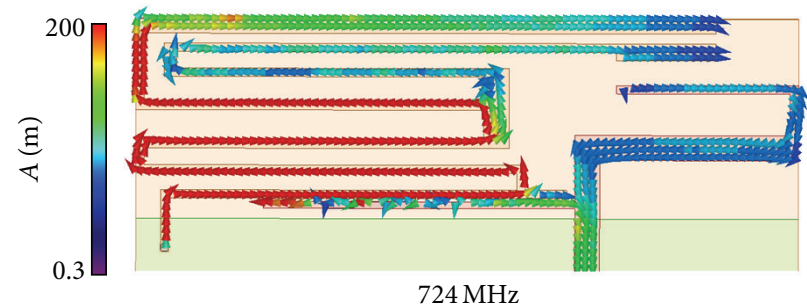

(a)

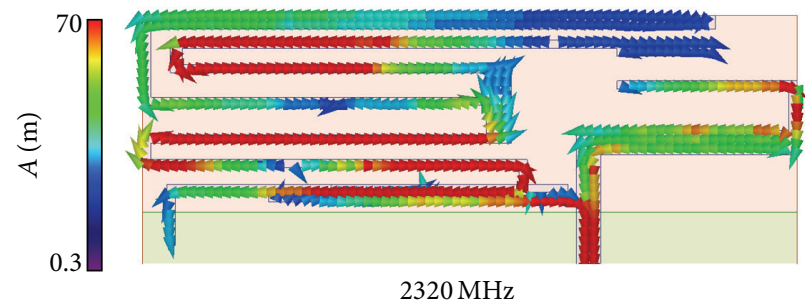

(c)

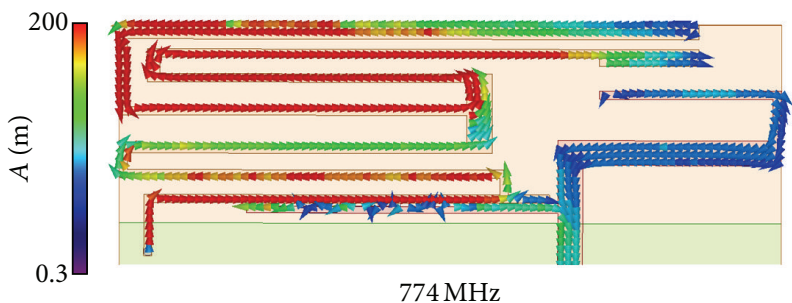

(b)

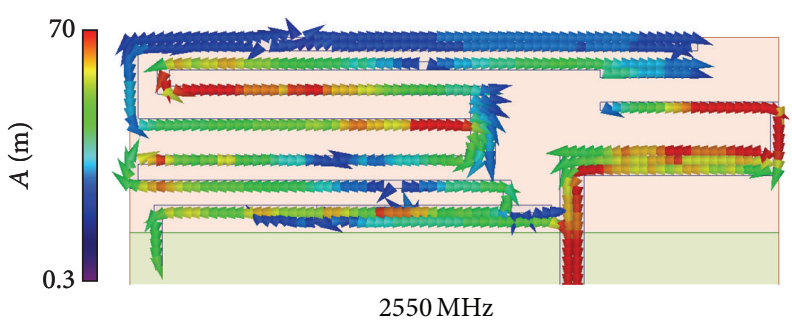

(d)

FIGURE 4: Simulated current distribution of the proposed miniaturized antenna.

The simulated reflection coefficient graph for the proposed antenna design when varying the parameter $L_{1}$ is shown in Figure 6. Since this path mainly generates the first resonant frequency $(724 \mathrm{MHz})$ of the required physical length of the resonator, we can observe a change in the resonant frequency points by changing the length $L_{1}$. Appropriate adjustment of this length can satisfy the low frequency band $(698 \mathrm{MHz})$ within the LTE700 operation band. In this paper, the optimal length for $L_{1}$ was $36 \mathrm{~mm}$. The simulated reflection coefficient graph when varying parameter $L_{2}$ is shown in Figure 7. The simulation result shows that when $L_{2}$ is changed, the second resonant frequency point of the low frequency band is changed. Therefore, appropriate control of the length $L_{2}$ can make the second resonant frequency point of the low frequency cover the high frequency points of the LTE700 operating bandwidth. In this paper, the optimal 


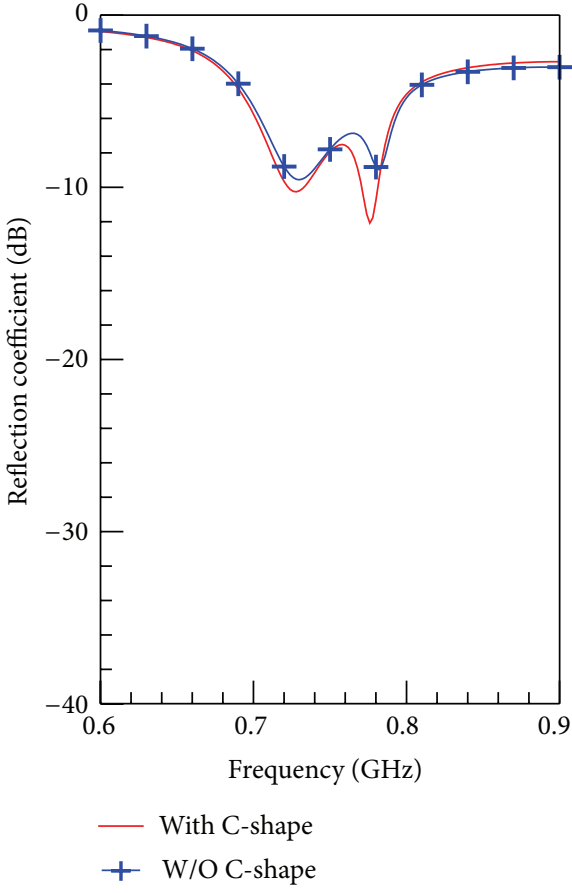

(a)

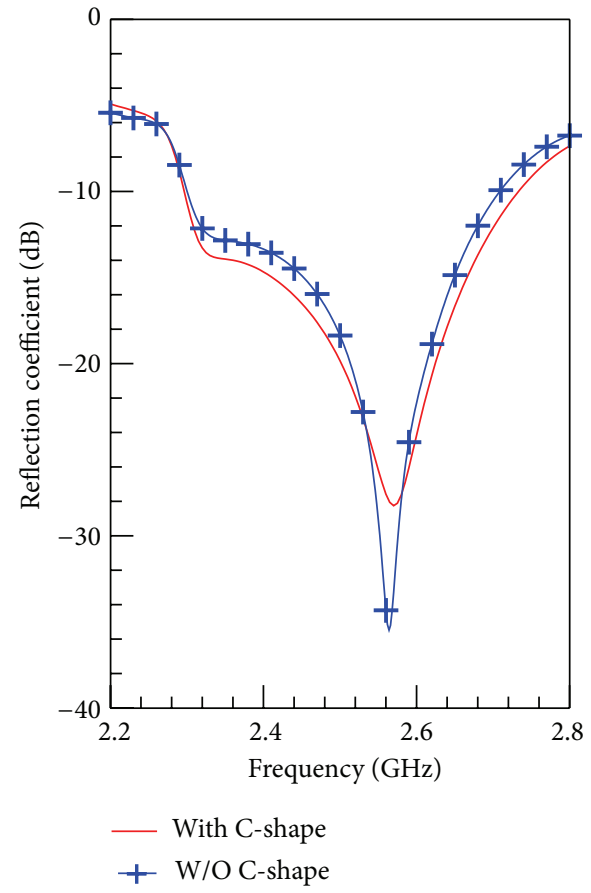

(b)

FIGURE 5: Simulated reflection coefficient of the proposed antenna with and without the C-shape coupler.

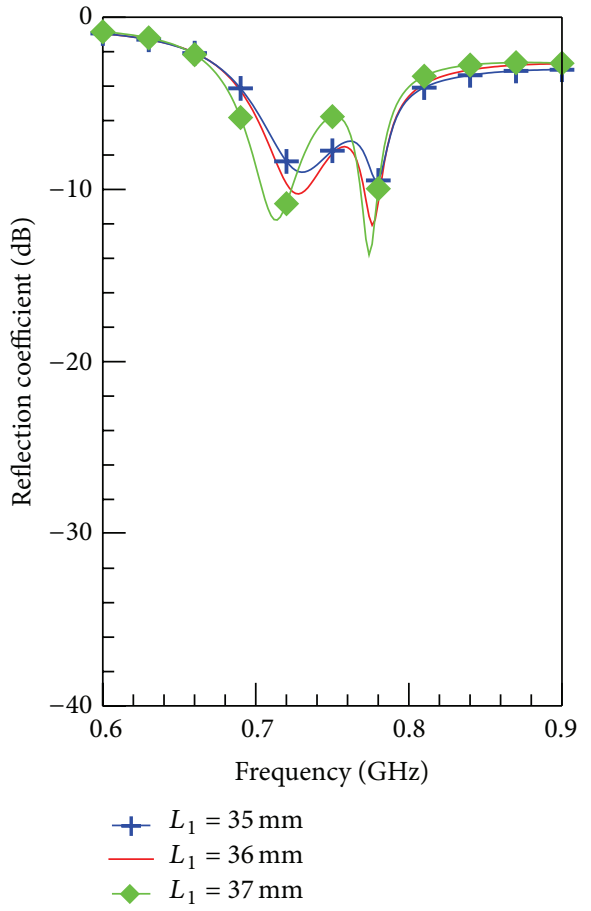

(a)

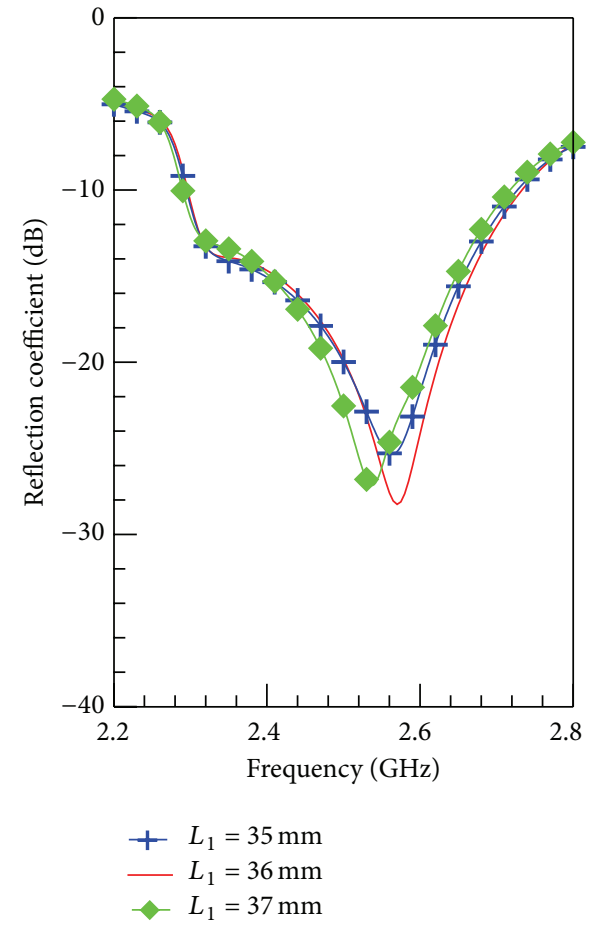

(b)

FIgURE 6: Simulated reflection coefficient of the proposed antenna as the function of $L_{1}$. 


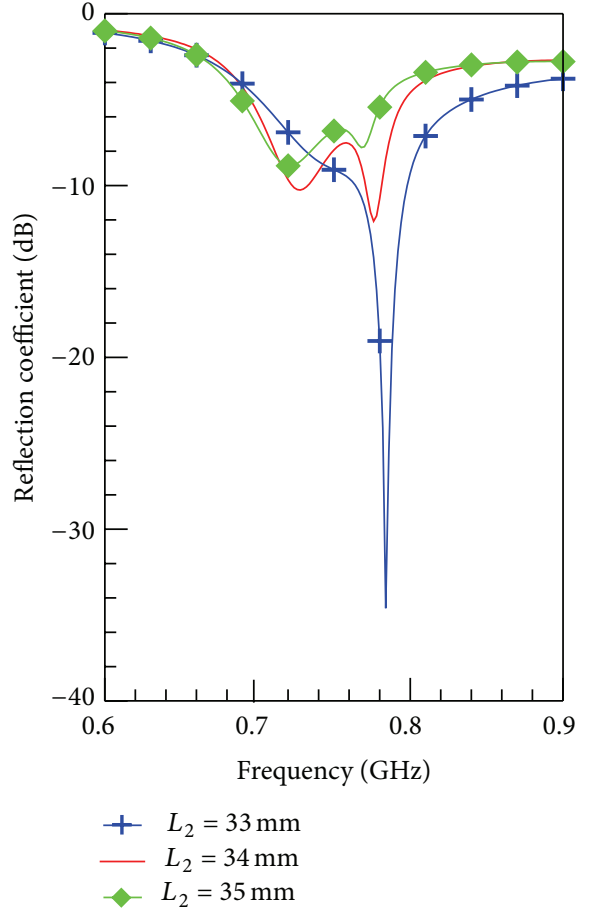

(a)

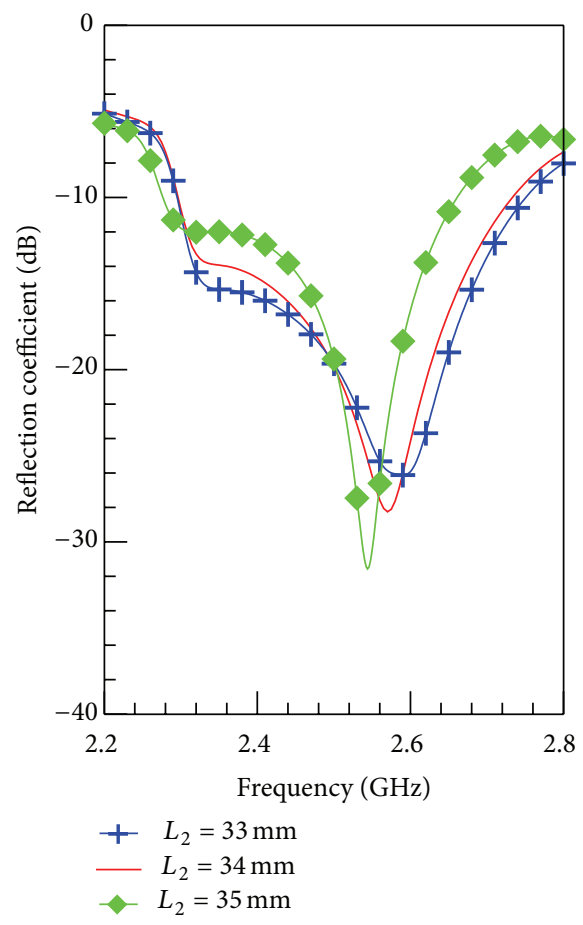

(b)

FIgURE 7: Simulated reflection coefficient of the proposed antenna as the function of $L_{2}$.

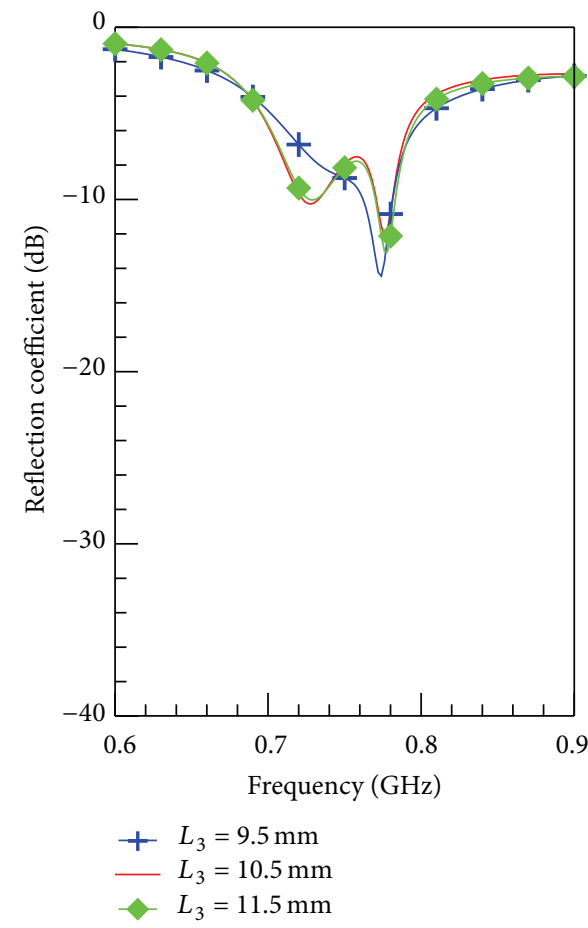

(a)

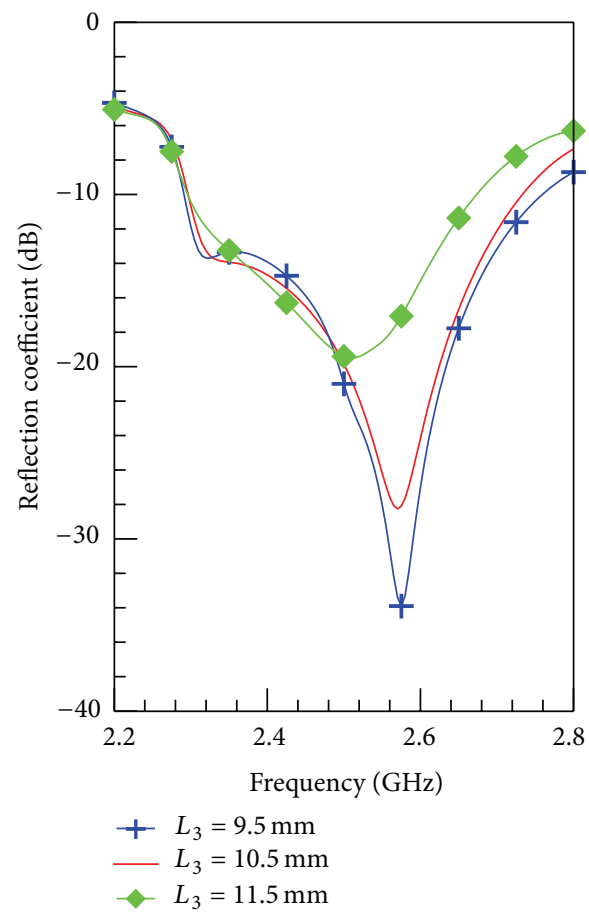

(b)

FIGURE 8: Simulated reflection coefficient of the proposed antenna as the function of $L_{3}$. 


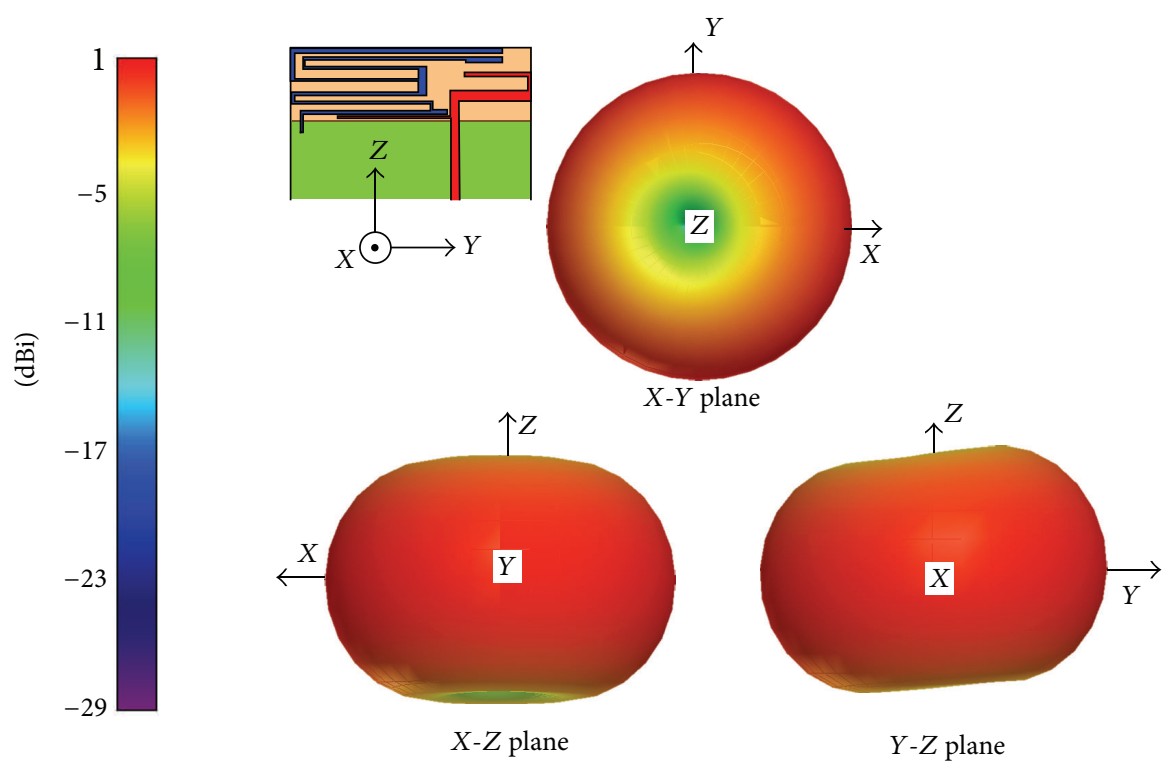

(a)
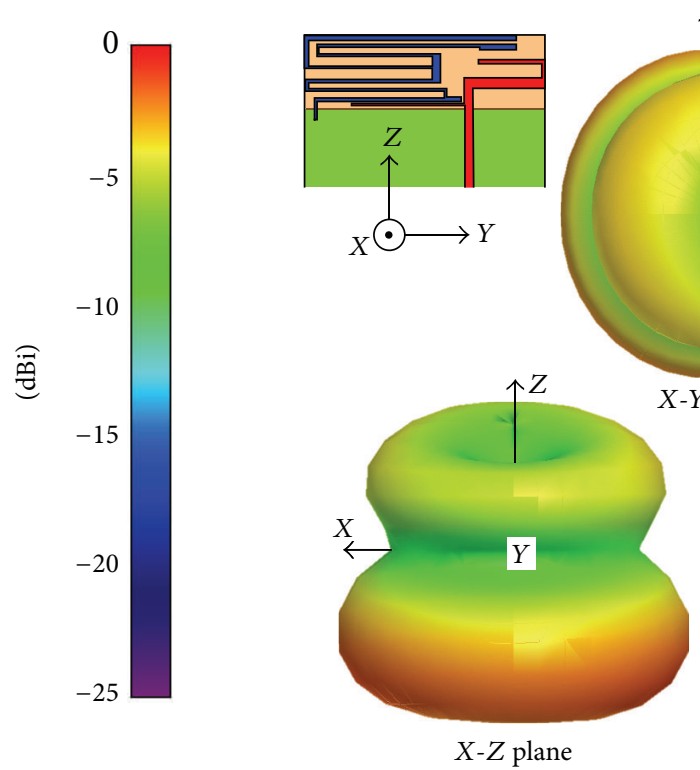

(b)

FIGURE 9: Simulated three-dimensional radiation pattern at (a) $750 \mathrm{MHz}$ and (b) $2500 \mathrm{MHz}$.

length for $L_{2}$ was $34 \mathrm{~mm}$. Appropriate control of the two parameters $L_{1}$ and $L_{2}$ ensures that low-frequency operation fully covers the bandwidth required for LTE700 operation. Through the embedding of the C-type coupler to improve the impedance matching at the junction, the two resonant frequencies can meet the full bandwidth operation requirement. In addition, because the high frequency operation of this antenna is the harmonic of the low-frequency resonant path, proper control of these two parameters can also make the high frequency cover the bandwidth required for LTE2300 operation.

The simulated reflection coefficient when varying the parameter $L_{3}$ is shown in Figure 8. The second harmonic for the high frequency operation is a monopole antenna with a $1 / 4 \lambda_{g}$ wavelength. We observe that as the length is changed, the resonant frequency varies. Therefore, appropriate control of the parameter can make the antenna operation fully cover the bandwidth required for LTE2500 operation. In the present work we design the antenna by adjusting the three parameters mentioned above, which can completely cover the range of the three LTE frequency bands.

3.3. Radiation Characteristics. The simulated and measured $3 \mathrm{D}$ radiation pattern of the proposed antenna design at low and high frequency is shown in Figures 9 and 10, respectively. The pattern in three different cuts is similar between the 


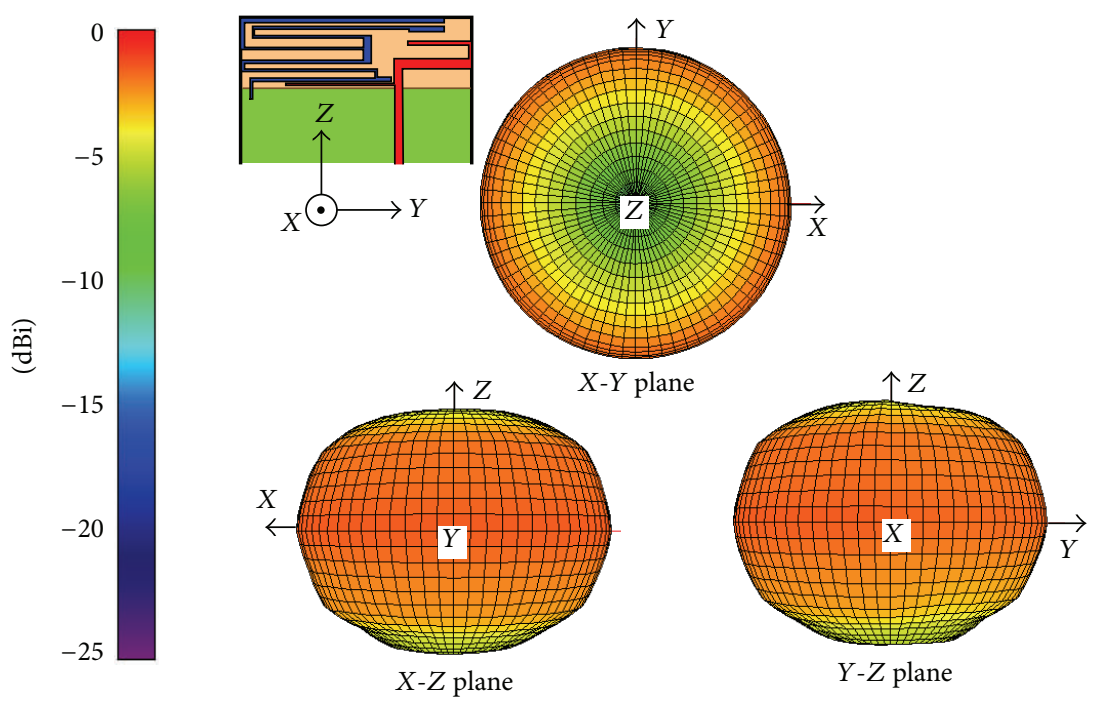

(a)

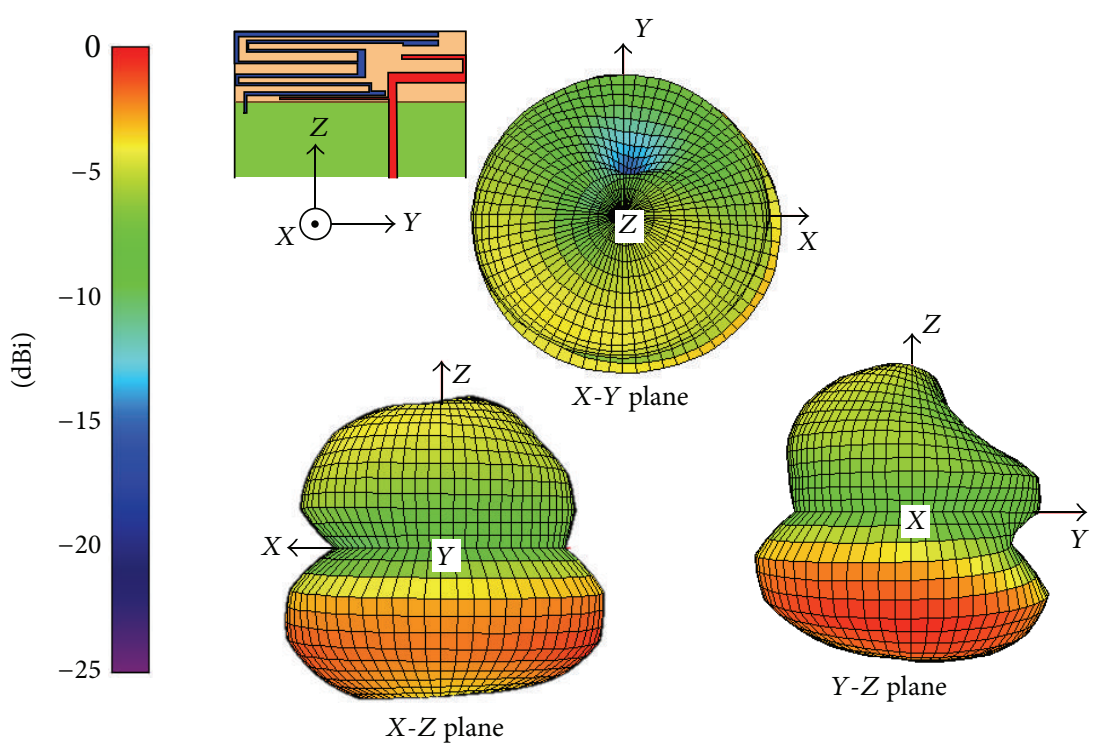

(b)

FIGURE 10: Measured three-dimensional radiation pattern at (a) $750 \mathrm{MHz}$ and (b) $2500 \mathrm{MHz}$.

simulated and measured results. In Figure 10(a) the measured $3 \mathrm{D}$ radiation pattern of the antenna at a central frequency of $750 \mathrm{MHz}$ is shown. A nearly omnidirectional radiation pattern is observed in the $X-Y$ plane. The characteristic of the radiation is ideal for handheld devices. The $3 \mathrm{D}$ radiation pattern of the designed antenna measured at $2500 \mathrm{MHz}$ is shown in Figure 10(b). Since the resonance mechanism at higher bands is a higher order mode, a null point could be observed at a higher frequency band. However, the radiation pattern remains in the horizontal section $(X-Y)$ with an omnidirectional radiation pattern, which is also conducive to handheld devices for wireless communication reception.

The measured antenna gain and efficiencies at low and high frequency are shown in Figures 11(a) and 11(b), respectively. In the former, the antenna gain variation is from $-0.05 \mathrm{dBi}$ to $-2.8 \mathrm{dBi}$ and the variation of the antenna efficiency ranges from $36.5 \%$ to $50.5 \%$. Since this antenna is a miniaturized antenna when operating in the low frequency band, the radiation efficiency is relatively unaffected by the limitation of the miniaturized design. However, in comparison to the reference design, the antenna presented here still meets LTE700 operational requirements with respect to gain and radiation when operating at low frequency. From Figure 11(b), the gain variation at the high frequency band is seen to vary from 3.4 to $2.1 \mathrm{dBi}$ while the antenna efficiency concurrently varies from $76.3 \%$ to $67.1 \%$. We can see that the proposed design is capable of stabilizing the antenna gain and efficiency. 


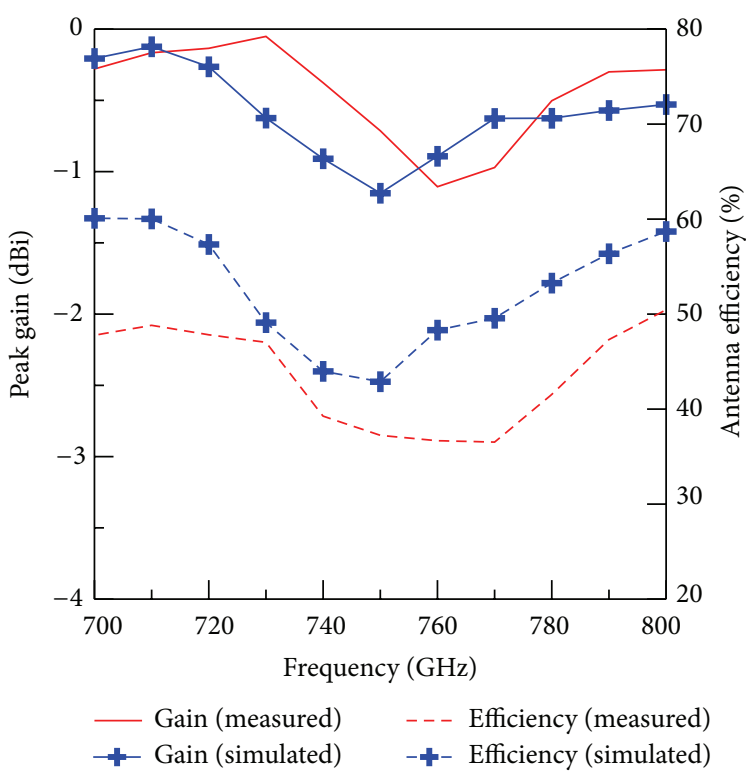

(a)

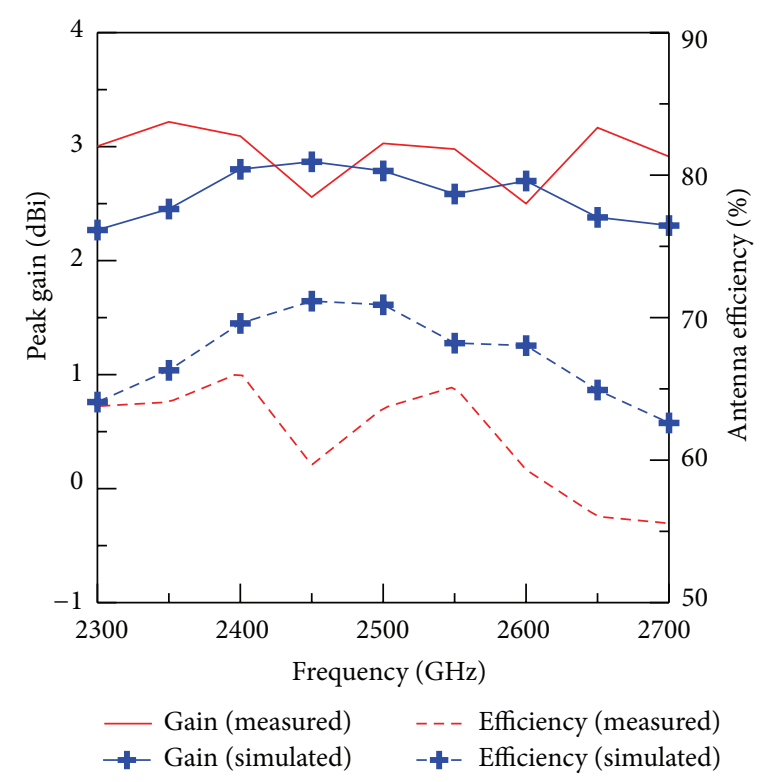

(b)

FIGURE 11: Measured and simulated antenna gain and antenna efficiency $\eta_{a}=\eta_{\mathrm{rad}} \cdot\left(1-\left|S_{11}\right|^{2}\right)$ of the proposed antenna versus frequency at (a) lower and (b) higher bands.

\section{Conclusion}

This paper proposes a multiband LTE antenna design for internal mobile device use. Since the antenna adopts a printed design, the actual production is cost effective and easily integrates with mobile systems. The antenna defined by the $-6 \mathrm{~dB}$ reflection coefficient at low frequency can resonate within a bandwidth of $110 \mathrm{MHz}(689 \sim 800 \mathrm{MHz})$. At high frequency under the $-10 \mathrm{~dB}$ reflection coefficient definition the antenna is capable of reaching a $410 \mathrm{MHz}(2.3 \sim 2.71 \mathrm{GHz})$ operational bandwidth. The antenna at low frequency utilizes two adjacent resonant frequencies to create a wider band and successfully uses a C-type coupling element to enhance impedance matching. Therefore, this antenna design can completely cover the required operational bandwidths of LTE700, LTE2300, and LTE2500. Our experimental results have shown that the antenna possesses favorable radiation characteristics irrespective of operation at low and high frequency bands.

\section{Conflict of Interests}

The authors declare that there is no conflict of interests regarding the publication of this paper.

\section{References}

[1] C.-W. Yang, Y.-B. Jung, and C. W. Jung, "Octaband internal antenna for 4G mobile handset," IEEE Antennas and Wireless Propagation Letters, vol. 10, pp. 817-819, 2011.

[2] M. S. Ahmad, C. Y. Kim, and J. G. Park, "Multishorting pins PIFA design for multiband communications," International
Journal of Antennas and Propagation, vol. 2014, Article ID 403871, 10 pages, 2014.

[3] P. Li, J. Pan, D. Yang, and P. Nie, "A novel dual-shorting point PIFA (GSM850 to IMT-A) for mobile handsets," International Journal of Antennas and Propagation, vol. 2013, Article ID 436808, 7 pages, 2013.

[4] L. Lizzi and A. Massa, "Dual-band printed fractal monopole antenna for LTE applications," IEEE Antennas and Wireless Propagation Letters, vol. 10, pp. 760-763, 2011.

[5] K.-L. Wong, C.-A. Lyu, and L.-C. Chou, "Small-size multiband planar antenna for LTE700/2300/2500 operation in the tablet computer," Microwave and Optical Technology Letters, vol. 54, no. 1, pp. 81-86, 2012.

[6] J. Lee, Y.-K. Hong, S. Bae, G. S. Abo, W.-M. Seong, and G.H. Kim, "Miniature long-term evolution (LTE) MIMO ferrite antenna," IEEE Antennas and Wireless Propagation Letters, vol. 10, pp. 603-606, 2011.

[7] K.-L. Wong and Y.-W. Chang, "Internal WWAN/LTE handset antenna integrated with USB connector," Microwave and Optical Technology Letters, vol. 54, no. 5, pp. 1154-1159, 2012.

[8] Ansoft High Frequency Structure Simulator (HFSS), Version 12. 0, Ansoft, 2009. 

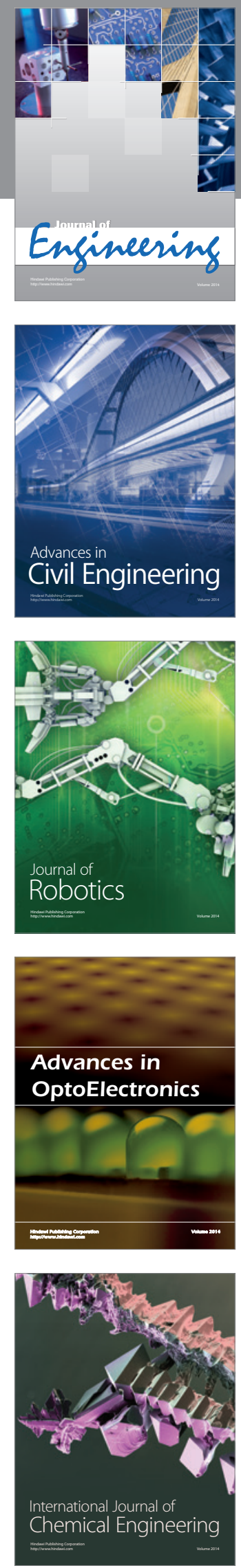

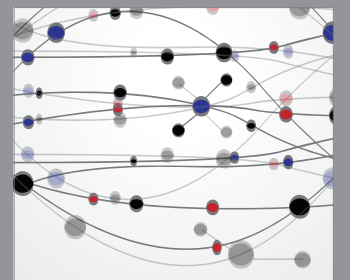

The Scientific World Journal
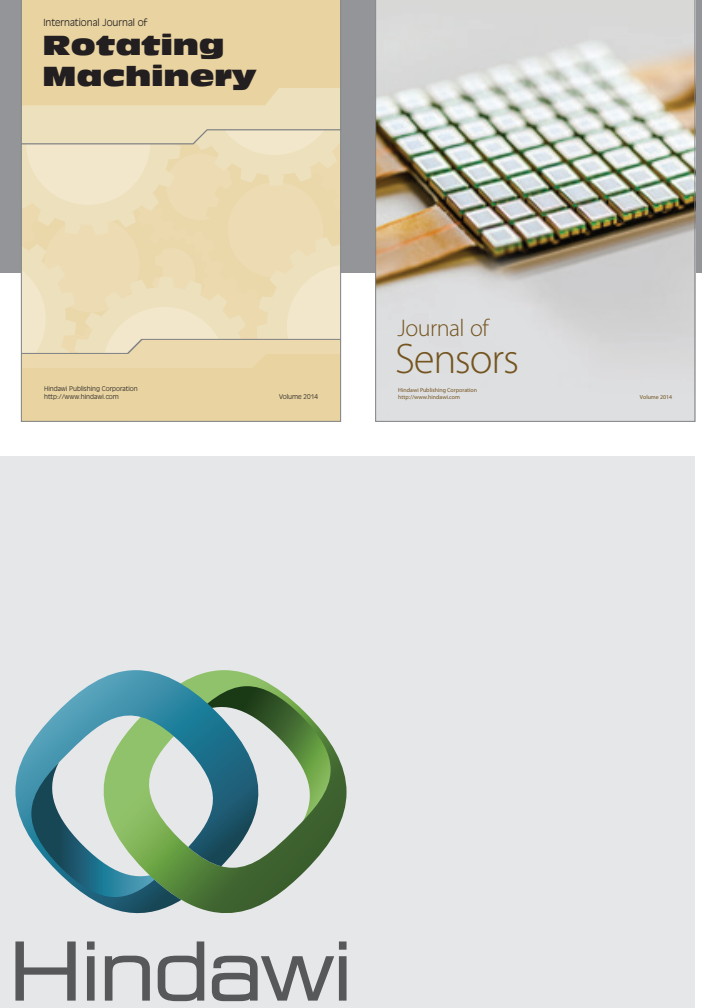

Submit your manuscripts at http://www.hindawi.com
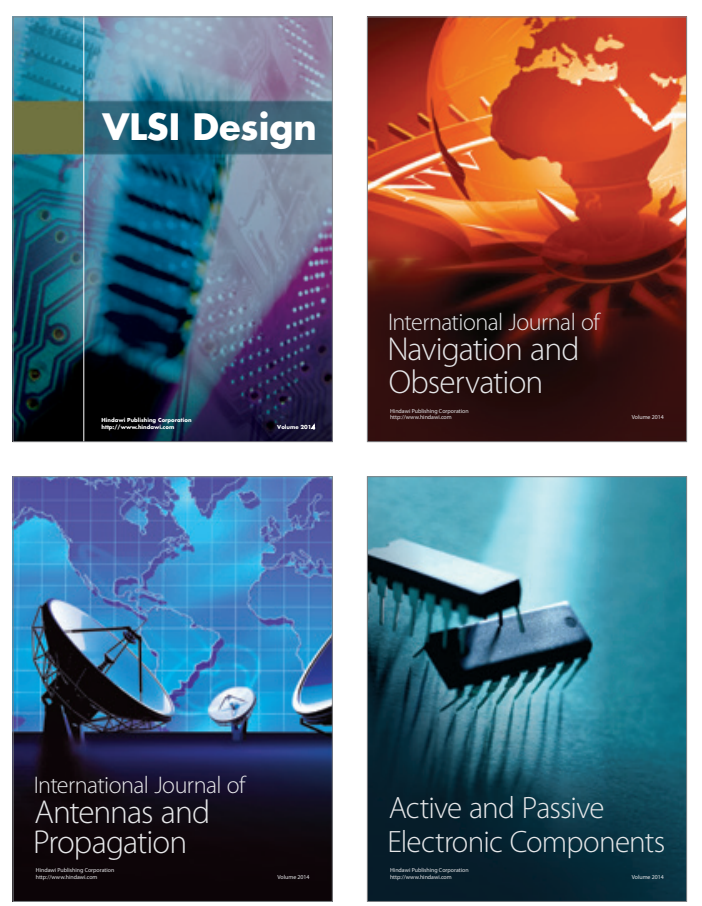
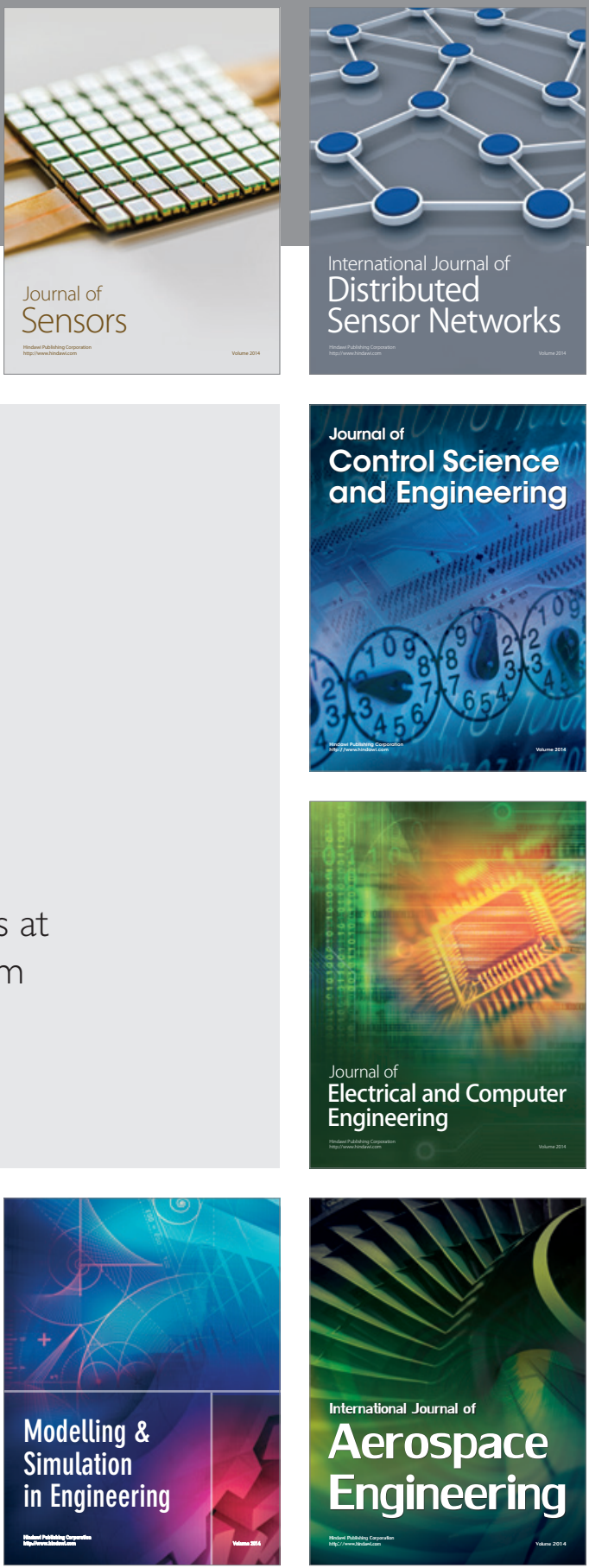

Journal of

Control Science

and Engineering
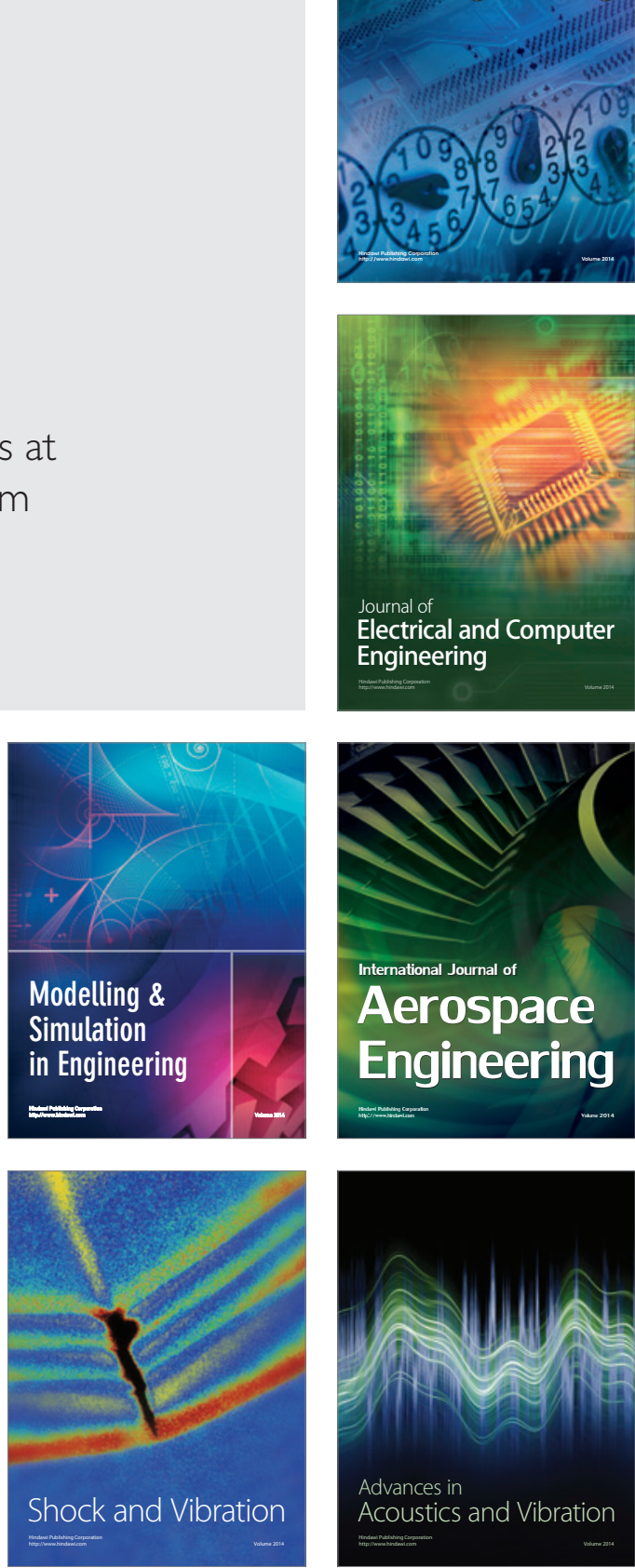